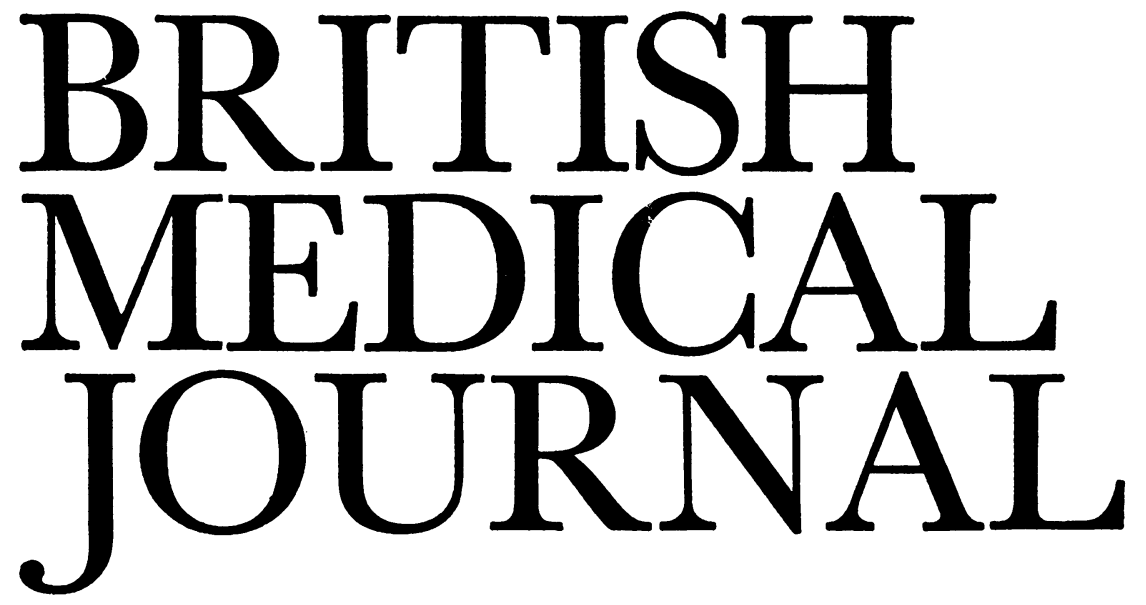

\title{
Erythema Multiforme
}

The term erythema multiforme means different things to different people. Unfortunately the word multiforme is often taken as a licence to apply it to almost any form of erythema of obscure origin. In some countries even such well-defined diseases as pemphigoid have been included. However, there is much to be said for using the diagnosis to describe only typical cases. It has recently been confirmed ${ }^{1}$ that such cases do also have a typical histology distinguishable from the other types of erythematous eruption.

Clinically, typical erythema multiforme can occur in various forms which differ in the severity of the cell damage, so that individual lesions may be macules, papules, or have superimposed blisters. The lesions also vary in number and distribution. Cases showing all types of lesion in any one attack, and cases showing the different clinical pictures in subsequent attacks, leave little doubt that they are all basically similar, though it can be argued that the most severe forms, or Stevens-Johnson syndrome, should be kept apart.

The mildest, papular or simplex, form of erythema multiforme is the commonest. The patient presents with dull red maculo-papules up to $2 \mathrm{~cm}$ in diameter, often showing the characteristic target lesions due to central stagnation or extravasation of red cells. These tend to have a typical distribution on the backs and fronts of the hands and the feet and on the extensor aspects of the elbows and knees, but may be more widespread. Occasionally they occur on mucous membranes. The differential diagnosis of this type must include drug eruptions; id eruptions from fungus, bacterial, or virus infections; and eruptions which are difficult to classify but which in appearance and natural history seem to be on the borderline between urticaria and erythema multiforme. One particular type of chilblain with multiple small lesions on the fingers may closely resemble erythema multiforme. ${ }^{2}$ Typical erythema multiforme may be associated with erythema nodosum and then requires the usual investigations for the latter to exclude tuberculosis, sarcoidosis, and streptococcal and other infections.

If the inflammatory process is more severe, blisters will form. Often the lesions are like the papular form, perhaps only few in number, but with superimposed blisters. In the most severe forms of the disease blistering may be very severe, with extensive mucosal involvement-the so-called Stevens-Johnson syndrome ${ }^{3}$ (there is some doubt whether the names of Stevens and Johnson are correctly attached to this syndrome ${ }^{4}$ ). Unlike the other types of erythema multiforme, in which constitutional upset is usually mild or none, this can be a very severe illness, making the patient extremely miserable, with high fever and with an appreciable mortality rate. Characteristically the mucous membrane of the mouth is almost entirely denuded of epithelium and the eyes show a severe conjunctivitis or corneal damage. Lesions on the genitalia may give rise to retention of urine. Renal disease occurs rarely. ${ }^{5}$ Bronchitis or pneumonitis, often more extensive on $x$-ray than suspected clinically, is commonly associated.

The differential diagnosis of the Stevens-Johnson syndrome includes Behçet's disease, pemphigus, pemphigoid, and toxic epidermal necrolysis or Lyell's disease. Behçet's disease should not cause confusion, as it essentially causes ulceration of the mouth rather than extensive erosions and is chronic. Pemphigus and pemphigoid are also chronic diseases with characteristic blisters, and any doubts can be cleared up by histological examination. Toxic epidermal necrolysis, with its typical scalded-skin appearance, should not cause confusion, though it was perhaps often classified with erythema multiforme before Lyell's description in 1956.6

The aetiology of all forms of erythema multiforme remains obscure in most cases. A variety of stimuli have been known to trigger it off, with a latent interval of one to three weeks. They include especially virus infections like herpes simplex and vaccinia, mycoplasma infections, drug sensitivities, pregnancy, and radiotherapy, but the list of reported causes is large. ${ }^{78}$ The most important drugs are barbiturates, long-acting sulphonamides, and phenylbutazone. $^{8-11}$ This all suggests that erythema multiforme is a pattern of reaction based on an immunological mechanism, though the exact type of this has yet to be demonstrated. Despite the occasional isolation of viruses or mycoplasmas from the blisters, ${ }^{12}$ it seems unlikely that there is a specific infective organism. There is no reason to regard these patients as being infectious, though they are often best looked after in isolation for their own protection.

From the practical point of view erythema multiforme in all its forms is a self-limiting disease over two to six weeks, and with the exception of drug sensitivities the initiating cause is often no longer active by the time the eruption 
has appeared. The disease runs much the same course whether a cause for it is found or not. The history and clinical examination must concentrate on drugs, infections, and analysis of any other symptoms. The urine should always be examined for protein and red cells. Biopsy is seldom necessary to establish the diagnosis and does nothing to help establish the underlying cause, though immunofluorescence studies may be helpful in the future. Skin testing has not been shown to be of any value, though occasional positive tests with herpes virus ${ }^{7}$ and other antigens have been reported. When drugs are suspected to be causing it they are best avoided in the future irrespective of any laboratory investigation. The danger that re-exposure will lead to one of the more severe forms of erythema multiforme does not warrant even giving test doses. Serological evidence of a preceding infection-for example, mycoplasma-may be found in an appreciable number of cases, but by the time the result is available it has usually become somewhat academic.

Treatment is essentially symptomatic. The milder forms of the disease require little apart from reassurance, and local applications cannot be expected to be much more than placebos. The ability of systemic steroids to reduce the mortality of the Stevens-Johnson syndrome has not been proved, but there is no doubt that they can sometimes provide dramatic symptomatic relief, with a starting dose of prednisone $60 \mathrm{mg}$ daily in a severe case. A systemic antibiotic is often combined to prevent secondary bacterial infection of the denuded area. Probably the most important part of treatment is skilled nursing. Recovery can be expected to be complete, with the possible exception of the more severe ocular complications.

Erythema multiforme sometimes recurs, but there is little that can be done to prevent recurrences apart from avoidance of offending drugs. Possibly in those cases that follow herpes simplex infection some success may attend early treatment of the herpes with idoxuridine.

\footnotetext{
${ }^{1}$ Ackerman, A. B., Penneys, N. S., and Clark, W. H., British fournal of

Dermatology, $1971,84,554$.
Haxthausen, H., Cold in Relation to Skin Diseases. Copenhagen, Levin and Munksgaard, 1930.

Ashby, D. W., and Lazar, T., Lancet, 1951, 1, 1091.

- Thomas, B. A., British Medical fournal, 1950, 1, 1393.

Comaish, J. S., and Kerr, D. N. S., British Medical fournal, 1961, 2, 84.

- Lyell, A., British Fournal of Dermatolcgy, 1956, 68, 355.

Shelley, W. B., fournal of the American Medical Association, 1967, 201, 153.

- Löffler, A., Jenny, P., and Zimmermann, H., Dermatologica (Basel), 1969, 138, Suppl. 5 .

- British Medical fournal, 1964, 2, 1410.

10 Carroll, O. M., Bryan, P. A., and Robinson, R. I., fournal of the American Medical Association, 1966, 95, 691 .

Medical Association, 1966, 95, 691.
1 Bianchine, J. R., et al., American fournal of Medicine, 1968, 44, 390.

12 Lyell, A., Gordon, A. M., Dick, H. M., and Sommerville, R. G., Lancet. $1967,2,1116$.
}

\section{Prophylaxis in Rheumatic Fever}

A striking feature of rheumatic fever is the frequency with which it recurs, and the rising incidence of valvular heart disease with each recurrence has been clearly shown. ${ }^{1}$ In 1940 M. G. Wilson ${ }^{2}$ reported that $85 \%$ of her patients had had a recurrence within eight years of the first attack. C. B. Perry $^{3}$ noted that $56 \%$ of his patients seen before 1939 had one or more recurrences, but after an intensive campaign for prophylaxis in 1955 there had been only one relapse among patients believed to be taking prophylaxis conscientiously. Florid rheumatic fever is now relatively rare and also less severe in the more economically advanced nations. ${ }^{4}$ Though this decrease in prevalence and severity began before the antibiotic era and has probably been associated with altered virulence of the streptococcus and improved social conditions, prompt and effective antibiotic therapy in group A streptococcal infections must have further lowered the incidence.

The changing clinical pattern of rheumatic fever, with minimal or no fever, often a non-migratory monarthritis, arthralgia, abdominal pain, or even carditis as the presenting feature, ${ }^{5}$ must be appreciated or it may be unrecognized and therefore untreated. A. R. Feinstein ${ }^{6}$ and Feinstein and M. Spagnuolo ${ }^{7}$ believed that recurrences of rheumatic fever tend to repeat the features of the first attack. They claimed that if the first attack did not affect the heart recurrences were also unlikely to do so, and they suggested that prophylaxis might be reserved for those who develop heart disease. Perry, ${ }^{3}$ however, was not so certain, and A. G. Kuttner and F. E. Mayer ${ }^{8}$ noted that $25 \%$ of cases did not follow a repetitive pattern. Nor does that take into account minimal carditis, which could easily be missed and which could be more severe in a second attack. Though the incidence of streptococcal infections tends to decrease with ageing the attack rate of rheumatic fever after an infection remains high in the susceptible.

Spagnuolo, B. Pasternack, and A. Taranta ${ }^{9}$ recently showed that symptoms of sore throat with fever were significantly associated with a high recurrence rate-up to $25 \%$ in children aged under 12 and $14 \%$ in adolescentsand particularly so when the interval since the preceding attack of rheumatic fever was short. Those with existing rheumatic heart disease and those who had had a number of previous attacks were also particularly susceptible to recurrences, but there was no significant association between recurrence and the rise in the antistreptolysin $\mathrm{O}$ titre. This means that prophylaxis is desirable in all children who have had rheumatic fever, while it is reasonable to instruct adults in a first attack without carditis to seek treatment promptly for any attack of sore throat.

Chemoprophylaxis, when decided on, should be with penicillin (penicillin V $120 \mathrm{mg}$ twice daily) or sulphadiazine $(0.5 \mathrm{~g}$ twice daily) by mouth, and should be given for a minimum of five years after an attack or indefinitely in cases of severe carditis. A sore throat occurring while on prophylaxis should be promptly treated with bactericidal doses of penicillin for 10 days. Spagnuolo and his colleagues ${ }^{9}$ stress the superiority in prophylaxis of a monthly injection of benzathine penicillin. The drawbacks of a monthly, painful injection have limited the use of benzathine penicillin in Britain. Allergic reactions to it are also relatively common. Oral prophylaxis conscientiously adhered to gives very reasonable protection, and, provided the severity and prevalance of rheumatic fever do not increase, there seems no reason to change British practice.

1 Biorck, G., fournal of Chronic Diseases, 1955, 1, 591.

2 Wilson, M. G., Rheumatic Fever Commonwealth Fund, London, 1940.

3 Perry, C. B., Annals of the Rheumatic Diseases, 1969, 28, 471.

4 Besterman, E., British Heart .ournal, 1970, 32, 579.

5 Feinstein, A. R., Bulletin on Rheumatic Diseases, 1966, 17, 423

6 Feinstein, A. R., and Spagnuolo, M., New England fournal of Medicine, $1960,262,533$.

7 Feinstein, A. R., and Spagnuolo, M., Medicine, 1962, 41, 279.

8 Kuttner, A. G., and Mayer, F. E., New England fournal of Medicine, 1963, 268, 1259 .

- Spagnuolo, M., Pasternack, B., and Taranta, A., New England fournal of Medicine, 1971, 285, 641 . 